\title{
EVALUATION OF THE ACCURACY OF DIGITAL MODELS OBTAINED FROM INTRAORAL SCANNERS WITH DIFFERENT CAD/CAM SCANNING TECHNOLOGIES- AN IN VITRO STUDY
}

\author{
Passent S. Aly, ${ }^{1}$ BDs, MSc 1, Cherif A. Mohsen, BDS, MSc,PhD 2.
}

ABSTRACT

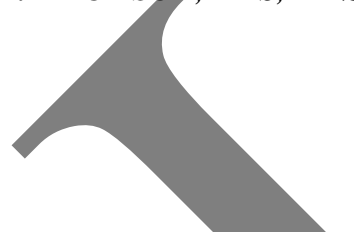

INTRODUCTION: An accurate digital impression is essential in fabrication of fixed dental prostheses. Digital dental impressions are recorded either by intraoral or extraoral scanners. Intraoral scanners scan abutments inside oral cavity eliminating the need of fabrication of dental casts. The extraoral scanners scan physical impressions or dental casts to produce a digital model.

OBJECTIVES: The aim of this study was to compare the accuracy of digital dental models produced by two different intraoral scanners in relation to the reference cast.

MATERIALS AND METHODS: This in vitro study inclurded an original typodont simulating patient's mouth. The reference typodont model was scanned by two intraoral scanners (3shape, Dental Wings) to produce digital models from both scanners. Mesiodistal (MD) and buccolingual (BL) measurements were recorded for canines, first premolars and first molars in both maxillary and mandibular jaws by same operator in the right and left sides and compared to measurements done on reference model. P values and adjusted means were calculated. Significance was set at $5 \%$. SPSS version 22.0 was used for statistical analysis.

RESULTS: There was no significant difference found between the accuracies of 3shape and Dental Wings digital models (p-value $=0.97)$ which were $<0.5 \mathrm{~mm}$, in comparison to the reference model. Regarding the reference teeth, the canine showed the highest accuracy ( $\mathrm{P}<0.0001)$.

CONCLUSIONS: The accuracy of digital models produced by the two intraoral scanners was similar with a minor level of discrepancies. Also, the canine showed the highest accuracy in the scanning procedure as it possess smooth surface.

KEYWORDS: Digital model; CAD/CAM; Intraoral scanner, Digital impression.

RUNNING TITLE: Accuracy of digital models by intraoral scanners.

1. Doctor, Department of Fixed Prosthodontics, Faculty of Dentistry, Minia University, Minia, Egypt.

2. Professor, Department of Fixed Prosthodontics, Faculty of Dentistry, Minia University, Minia, Egypt.

*Corresponding author:

Email:passentaly@yahoo.com

INTRODUCTION

Digital 3D dental models had a positive impact in the dental field as in prosthodontics they can be used for diagnosis, treatment planning, virtual alignment of dental implants and fabrication of final prosthetic restorations $(1,2)$. Also it is efficient in orthodontics eliminating the need of physical study casts that are used in space analysis and occlusion examination (3).

Digital dental scanners are classified into intraoral and extraoral scanners (4). Extraoral scanners are used for scanning physical dental impressions or stone casts so conventional impression procedure is still mandatory $(5,6)$. That's why intraoral scanners are more preferred over extraoral scanners in the dental procedures. Intraoral scanners are used for scanning prepared teeth, implant scan bodies and gingival tissues directly from the patient's mouth (5). They eliminate the use of conventional impression material reducing the occurrence of patient's vomiting reflux, discomfort and the unfavorable taste. Also, they avoid the need of impression disinfection, impression distortion,

fracture or abrasion of stone casts and the need of large storage area for patients' casts $(5,7)$. Where In addition, intraoral scanners allow the dentist to visualize the tooth preparation, adjust it if needed and rescan in the same visit reducing patient's visits to the dental clinic (7).

However, the accuracy of intraoral scanners are affected by the presence of blood or saliva inside the oral cavity as well as patient's limited mouth opening that might result in the production of an inaccurate digital impression (8).

Nevertheless it was proven in previous studies that intraoral scanners provide sufficient system accuracy for clinical application. That's why nowadays, intraoral scanners are widely used to import scan data for the fabrication of dental restorations $(9,10)$.

The quality and longevity of dental restorations have been enhanced after the use of CAD/CAM technology in dental field as the use of machined ceramic restorations eliminated the presence of voids, cracks or fracture lines that may occur in the conventional fabrication techniques (9). 
The aim of the present study was to evaluate the accuracy of digital models produced by two intraoral scanners (3shape and Dental wings). The null hypothesis was that there was no difference in the accuracy of the digital models produced by the two used intraoral scanners.

\section{MATERIALS AND METHODS}

An original typodont (ModuPRO Pros, Acadental Inc, Overland Park, KS) with full set of maxillary and mandibular teeth was used as a reference model. The maxillary and mandibular typodont jaws were scanned by two intraoral scanners (Trios 3shape, Dental Wings) producing 3shape and Dental Wings digital models as in figure 1.
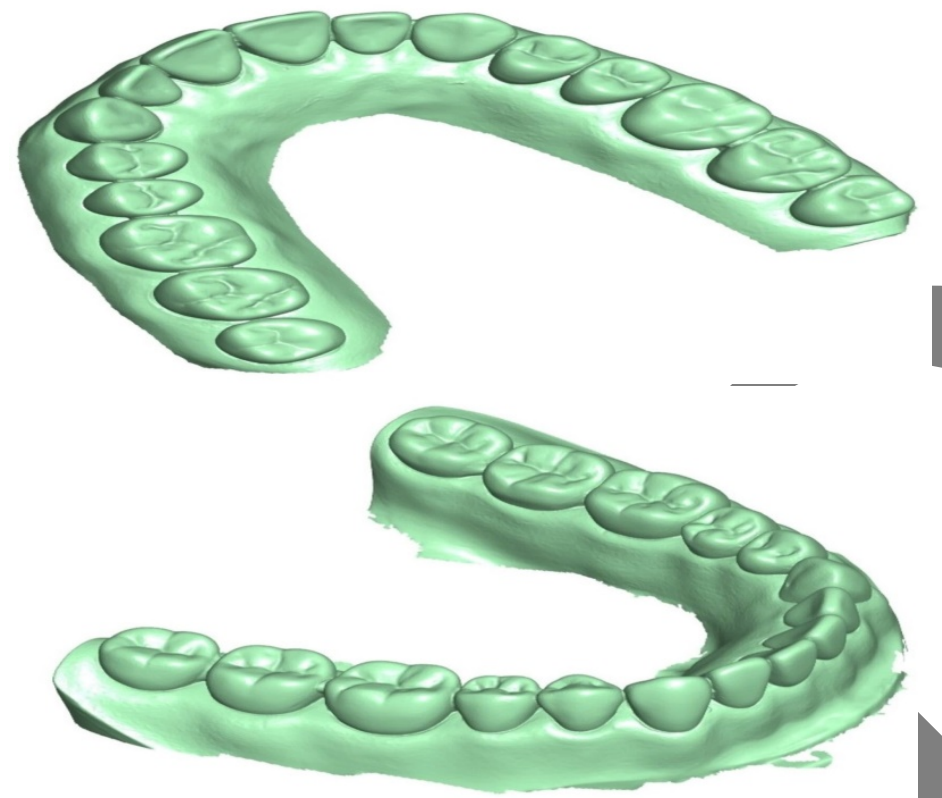

Figure 1: Scanned upper and lower casts.

Scanning technique of maxillary jaw started from the distal surface of the maxillary second right molar extended occlusally, buccally, linguallyand mesially then continued in same sequence for each tooth ending in the distal surface of maxillary second left molar. The same was done on the mandibular jaw where the head of scanner was held at an angle of 45 degrees to the long axis of the tooth. Scanning of each jaw was recorded in two minutes.

Measurements

The reference teeth (canines, 1st premolars and 1st molars) on each digital model were measured on desktop screen by Geomagic Studio 5 software (Raindrop Geomagic, Inc, Morrisville, NC) and the measurements were recorded from the screen by the program caliper. Measurements on the reference model (control) were done by one operator using a digital caliper (series 500 Digimatic ABSolute Caliper, Mitutoyo Corporation, Kawasaki, Japan). The reference cast was scanned ten times by each scanner producing ten 3shape and ten Dental Wings digital models. These measurements were repeated twice more, at least 1 week apart by the same operator.

Buccolingual (BL) and Mesiodistal (MD) measurements were recorded in an occlusal direction for each reference tooth in the digital models and reference typodont model. BL measurements were recorded from the middle of the buccal surface to the middle of the lingual surface. While MD measurements were recorded from the mesial contact area (center of the mesial surface) to the distal contact area (center of the distal surface). Discrepancy recorded less than $0.5 \mathrm{~mm}$ $(11,12)$ was considered clinically acceptable.

\section{Statistical Analysis:}

Univariate general linear regression model was used to evaluate factors affecting scanning accuracy: (1) type of scanner (3shape and Dental Wings intraoral scanners), (2) tooth (canines, premolars or molars) and (3) measurement direction (BL or MD). Descriptive statistics of the study groups were calculated, including means and standard deviations. P-values less than 0.05 were considered statistically significant.

\section{RESULTS}

Table 1 shows that there was no significant difference between scanners in the discrepancies $(\mathrm{P}=0.97)$. All errors were $<0.5 \mathrm{~mm}$. Also regarding tooth and direction of measurement, no significant differences in discrepancies were recorded $(\mathrm{P}=0.33,0.06)$ respectively.

Table 1: Factors affecting the differences between digital measurements of the 2 scanners and the reference cast.

\begin{tabular}{|l|l|l|}
\hline Factors & F & P value \\
\hline Type of scanner & 0.09 & 0.97 \\
\hline Tooth & 0.96 & 0.33 \\
\hline Direction of measurement & 3.55 & 0.06 \\
\hline
\end{tabular}

*: statistically significant at $\mathrm{P}<0.05$

Figures 2-3 show the discrepancies between the digital measurements of the two scanners and the reference cast in $\mathrm{BL}$ and $\mathrm{MD}$ directions. The MD and BL discrepancies were all $< \pm 0.3 \mathrm{~mm}$.

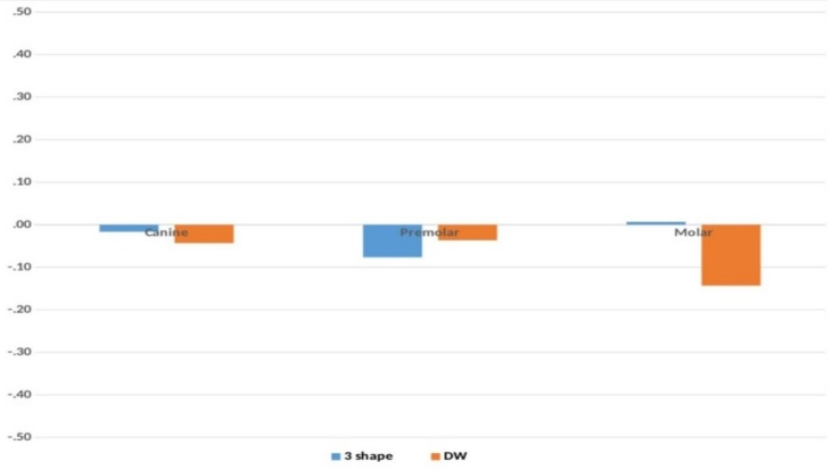

Figure 2: Differences of bucco-lingual digital measurements relative to the reference cast (in $\mathrm{mm}$ ) of the 2 scanners.

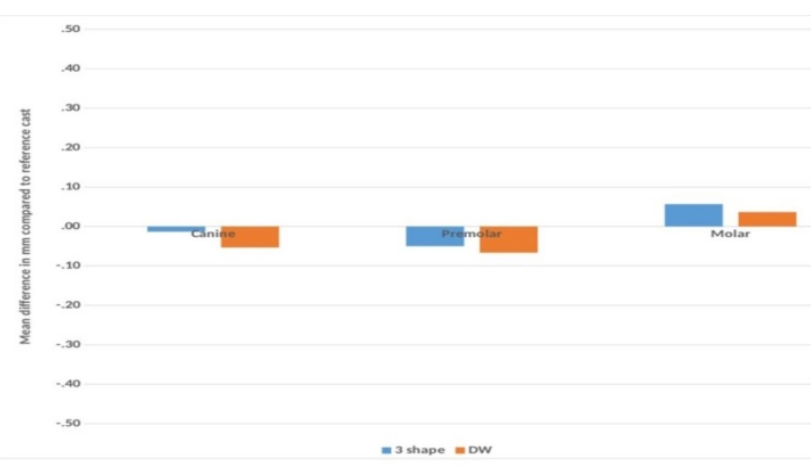

Figure 3: Differences of mesio-distal digital measurements relative to the reference cast (in $\mathrm{mm}$ ) of the 2 scanners. 
Figure 4 shows the adjusted mean discrepancies of both scanners. There was a higher underestimation in premolars than canines measurements (adjusted means $=-0.03$ and -0.02 $\mathrm{mm}$ ) in comparison to the reference teeth while in case of molars measurements there was an overestimation (adjusted mean= $0.1 \mathrm{~mm}$ ).

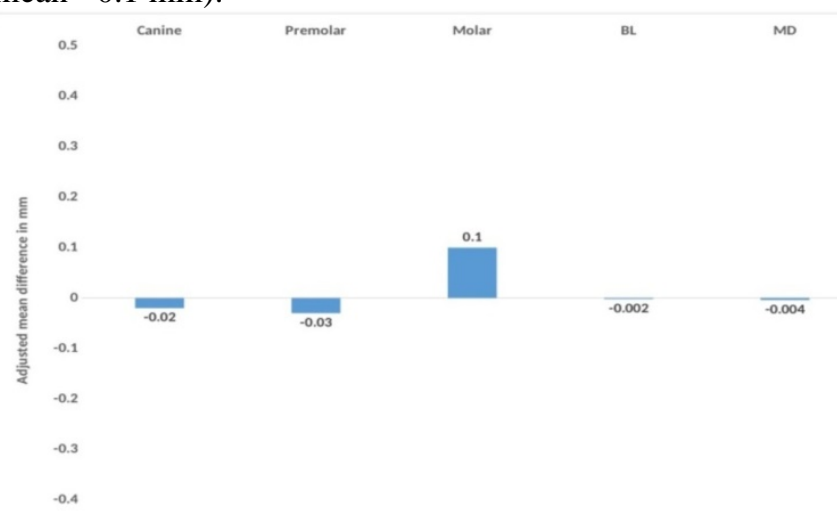

Figure 4: Adjusted mean differences between digital measurements and reference cast by tooth type and measurement aspect.

\section{DISCUSSION}

In digital dentistry, 3D digital model is produced by the use of either intraoral or extraoral scanning technology. Each type of scanner has its own advantages and disadvantages but still the studies didn't favor the use of certain type of scanner over the other one (13). This study evaluated the accuracy of different intraoral scanners in producing digital models using CAD/CAM technology. The null hypothesis was accepted as the accuracy of digital models produced by the two intraoral scanners was nearly similar.

The results of the current study showed that $\mathrm{BL}$ and $\mathrm{MD}$ measurements of both intraoral scanners expressed minor discrepancy but with no significant difference which proved that the two intraoral scanners used possess similar accuracy in producing digital models with an equivalent dimensions to that of the reference model.

This result is in coincidence with Ender and Mehl (14) study which proved that the accuracy of digital impression and conventional impression is similar. But they suggested making further studies to confirm their results.

The results verified the ability of using digital models as a successful replacement for the conventional stone model in dentistry. Several previous studies have proven that there was no statistically significant difference between measurements recorded from digital dental models and conventional stone models which comes in agreement with our results $(15,16)$. Also, Wiranto et al.(17) and Naidu and Freer (18) studies reported that measurements obtained from digital models by intraoral scanners were similar to that obtained from stone models although our study was done in vitro.

While other studies found a significant difference between digital and conventional stone models but was within the clinically acceptable range $(19,20)$. As it was recorded in several studies that discrepancy less than $0.5 \mathrm{~mm}$ are not counted as a significant deviation. $(11,12)$

But in Syrek et al. (21) in vivo study, it was reported that intraoral scanner Lava produced accurate restorations more than that produced from conventional procedures. This result was similar to our results regarding the high accuracy of introral scanners although the digital model produced is from a different intraoral scanner and compared to conventional stone cast not reference cast as in our study.

In the current study the digital models showed slight underestimation in case of canines and overestimation in case of molars but within clinically acceptable range. Canine showed the highest accuracy in comparison to premolars and molars. This bias might be due to the smooth surface of canine with no any irregularities or grooves as in premolars and molars (22). Also, it might be attributed to the different scanning technology, type of scanner, scanning position and oral cavity factors such as saliva, blood, limited mouth opening and difficulty in accessibility to posterior teeth as mentioned in previous studies. (23-25)

Im et al. (26) and Reuschl et al. (27) studies reported that the mesiodistal measurements of most teeth were underestimated in the digital model compared to those in a cast model. This comes partly in agreement with our results regarding premolars and canines but not in agreement with molars results. The difference might be referred to the use of other types of intraoral scanners that need application of titanium oxide powder on teeth for more accurate scanning. (28) Finally, Alcan et al (29) concluded that virtual measurements on digital casts were more accurate and less time consuming than using digital caliper on conventional stone models, and this caused the use of digital models to expand in the prosthodontic field $(15,19)$.

The cost of digital impression systems is still a major issue. Christensen (30) suggested that the relative cost of digital versus elastomer impressions will depend on the number of impressions that a dentist takes per month. A digital impression system has to be used for a significant number of patients to compensate for its cost.

\section{Limitations:}

This study is an in vitro study and is restricted to two types of intraoral scanners. Further in vivo studies are needed to be done inside the patient's mouth to simulate the oral environment such as saliva, blood and patient's tongue movement during recording the impression. Also, other types of intraoral scanners and extraoral scanners need to be studied in future studies.

\section{CONCLUSIONS}

Within the limitations of this in vitro study, it can be concluded that:

1. All digital models have a slight discrepancy, but this minor discrepancy is within the clinically acceptable range so would not restrict their clinical use in dentistry.

2. Recording the digital mesial-distal (MD) and bucco-lingual (BL) measurements is the best combination of accuracy, time saving, and therefore the best choice for routine digital measurements.

3. There is no statistically significant difference in accuracy of digital models produced by the two intraoral scanners used.

4. Canines showed highest accuracy in comparison to premolars and molars due to the smoothness of canine surface without any grooves or concavities as in premolars and molars. 


\section{Conflict of interest:}

The authors declare that they have no conflicts of interest.

\section{Acknowledgments}

The authors thank Prof. Maha El Tantawi for her support in terms of statistical analysis.

\section{REFERENCES}

1. Bentz RM, Balshi SF. Complete oral rehabilitation with implants using CAD/CAM technology, stereolithography, and conoscopic holography. Implant Dent. 2012;21:8-12.

2. El Kerdani $T$, Roushdy $\mathrm{S}$. The Use of CAD/CAM Technology for Fabricating Cast Gold Survey Crowns under Existing Partial Removable Dental Prosthesis. A Clinical Report. J Prosthodont. 2017;26:321-6.

3. Leifert MF, Leifert MM, Efstratiadis S, Cangialosi T. Comparison of space analysis evaluations with digital models and plaster dental casts. Am J Orthod Dentofacial Orthop 2009;136:16.

4. González de Villaumbrosia P, Martínez-Rus F, GarcíaOrejas A, Salido MP, Pradíes G. In vitro comparison of the accuracy (trueness and precision) of six extraoral dental scanners with different scanning technologies. J Prosthet Dent. 2016;116:543-50.

5. Guth JF, Keul C, Stimmelmayr M, Beuer F, Edelhoff D. Accuracy of digital models obtained by direct and indirect data capturing. Clin Oral Investig. 2013;17:1201-8.

6. Flügge TV, Schlager S, Nelson K, Nahles S, Metzger MC. Precision of intraoral digital dental impressions with iTero and extraoral digitization with the iTero and a model scanner. Am J Orthod Dentofacial Orthop. 2013;144:471-8.

7. Renne W, Ludlow M, Fryml J, Schurch Z, Mennito A, Kessler R, et al. Evaluation of the accuracy of 7 digital scanners: An in vitro analysis based on 3-dimensional comparisons. J Prosthet Dent. 2017;118:36-42.

8. Rudolph H, Salmen H, Moldan M, Kuhn K, Sichwardt V, Wostmann B, et al. Accuracy of intraoral and extraoral digital data acquisition for dental restorations. J Appl Oral Sci. 2016; 24:85-94.

9. Persson A, Andersson M, Oden A, Sandborgh-Englund G. A three-dimensional evaluation of a laser scanner and a touch-probe scanner. J Prosthet Dent 2006;95:194-200.

10. Hewlett E, Orro M, Clark G. Accuracy testing of threedimensional digitizing systems. Dent Mater 1992;8:49-53.

11. Hassan WN, Yusoff Y, Mardi NA. Comparison of reconstructed rapid prototyping models produced by 3dimensional printing and conventional stone models with different degrees of crowding. Am J Orthod Dentofacial Orthop 2017;151:209-18

12. Hazeveld A, Slater JJ, Ren Y. Accuracy and reproducibility of dental replica models reconstructed by different rapid prototyping techniques. Am J Orthod Dentofacial Orthop 2014;145:108-15.

13. Bohner LOL, De Luca Canto G, Marció BS, Laganá DC, Sesma N, Tortamano Neto P. Computer-aided analysis of digital dental impressions obtained from intraoral and extraoral scanners. J Prosthet Dent. 2017;118:617-23.

14. Ender A, Mehl A. Full arch scans: conventional versus digital impressions, an in-vitro study. Int $\mathrm{J}$ Comput Dent 2011;14:11-21.25. 26
15. Bell A, Ayoub AF, Siebert P. Assessment of the accuracy of a three-dimensional imaging system for archiving dental study models. J Orthod. 2003;30:219-23.

16. Gracco A, Buranello M, Cozzani M, Siciliani G. Digital and plaster models: a comparison of measurements and times. Prog Orthod. 2007;8:252-9.

17. Wiranto MG, Engelbrecht WP, Tutein Nolthenius HE, van der Meer WJ, Ren Y. Validity, reliability, and reproducibility of linear measurements on digital models obtained from intraoral and cone-beam computed tomography scans of alginate impressions. Am J Orthod Dentofacial Orthop 2013; 143: 140-7.

18. Naidu D, Freer TJ. Validity, reliability, and reproducibility of the iOC intraoral scanner: A comparison of tooth widths and Bolton ratios. Am J Orthod Dentofacial Orthop 2013; 144: 304-10.

19. Mullen SR, Martin CA, Ngan P, Gladwin M. Accuracy of space analysis with e models and plaster models. Am J Orthod Dentofacial Orthop. 2007;132:346-52.

20. Zilberman O, Huggare JA, Parikakis KA. Evaluation of the validity of tooth size and arch width measurements using conventional and three-dimensional virtual orthodontic models. Angle Orthod. 2003;73:301-6.

21. Syrek A, Reich G, Ranftl D, Klein C, Cerny B, et al. Clinical evaluation of all-ceramic crowns fabricated from intraoral digital impressions based on the principle of active wavefront sampling. J Dent. 2010; 38:553-9.

22. Persson ASK, Andersson M, Odén A, SandborghEnglund G. Computer aided analysis of digitized dental stone replicas by dental CAD/CAM technology. Dent Mater. 2008;24:1123-30

23. Flügge TV, Schlager S, Nelson K, Nahles S, Metzger MC. Precision of intraoral digital dental impressions with iTero and extraoral digitization with the iTero and a model scanner. Am J Orthod Dentofacial Orthop 2013;144, 471-478.

24. Rudolph H, Salmen H, Moldan M, Kuhn K, Sichwardt V, Wöstmann B et al. Accuracy of intraoral and extraoral digital data acquisition for dental restorations. J Appl Oral Sci 2016;24, 85-94.

25. Güth JF, Runkel C, Beuer F, Stimmelmayr M, Edelhoff D, Keul C. Accuracy of five intraoral scanners compared to indirect digitalization. Clin Oral Investig 2017;21,1445-55. 26. Im J, Cha JY, Lee KJ, Yu HS, and Hwang CJ. "Comparison of virtual and manual tooth setups with digital and plaster models in extraction cases," Am J Orthod Dentofacial Orthop. 2014;145:434-42.

27. Reuschl RP, Heuer W, Stiesch M, Wenzel D, and Dittmer MP. "Reliability and validity of measurements on digital study models and plaster models,” Eur J Orthod. 2016;38:22-6. 28. das Neves FD, do Prado CJ, Prudente MS, Carneiro TA, Zancope K, Davi LR, et al. Microcomputed tomography marginal fit evaluation of computer-aided design/computeraided manufacturing crowns with different methods of virtual model acquisition. Gen Dent 2015;63:39-42.

29. Alcan T, Ceylanoglu C, Baysal B. The relationship between digital model accuracy and time-dependent deformation of alginate impressions. Angle Orthod 2009;79:30-6.

30. Christensen G. Digital dentistry: Is this the future of dentistry? dental economics.com, 2011. 